\title{
Enhanced inhibition of Avian leukosis virus subgroup J replication by multi-target miRNAs
}

\author{
Qing-Wen Meng ${ }^{*}$, Zai-Ping Zhang, Wei Wang, Jin Tian and Zhi-Guang Xiao
}

\begin{abstract}
Background: Avian leukosis virus (ALV) is a major infectious disease that impacts the poultry industry worldwide. Despite intensive efforts, no effective vaccine has been developed against ALV because of mutations that lead to resistant forms. Therefore, there is a dire need to develop antiviral agents for the treatment of ALV infections and RNA interference (RNAi) is considered an effective antiviral strategy.

Results: In this study, the avian leukosis virus subgroup J (ALV-J) proviral genome, including the gag genes, were treated as targets for RNAi. Four pairs of miRNA sequences were designed and synthesized that targeted different regions of the gag gene. The screened target (i.e., the gag genes) was shown to effectively suppress the replication of ALV-J by $19.0-77.3 \%$. To avoid the generation of escape variants during virus infection, expression vectors of multi-target miRNAs were constructed using the multi-target serial strategy (against different regions of the gag, pol, and env genes). Multi-target miRNAs were shown to play a synergistic role in the inhibition of ALV-J replication, with an inhibition efficiency of viral replication ranging from 85.0-91.2\%.
\end{abstract}

Conclusion: The strategy of multi-target miRNAs might be an effective method for inhibiting ALV replication and the acquisition of resistant mutations.

Keywords: ALV, miRNA, Inhibition, Gag, Multi-target series

\section{Background}

Avian leukosis (AL) is the general term for a variety of neoplastic diseases of poultry caused by the Alpharetrovirus, Avien leukosis virus (ALV). ALV has been classified into 10 subgroups, designated A-J. The subgroup J virus (ALV-J) is a relatively new strain of ALV that was isolated from Dorking fowl in the early 1990s [1]. ALV is an RNA virus with a genome of approximately $7.6 \mathrm{~kb}$. The proviral genome of ALV-J contains three major genes, gag, pol, and env, which encode the viral structural proteins, RNA-dependent DNA polymerase, and the envelope glycoprotein, respectively.

RNA interference (RNAi) is a simple and effective tool for silencing target genes that involves endogenous or exogenous double-stranded RNA (dsRNA)-mediated degradation of the specific mRNA sequences. The main nucleic acid molecules that induce gene silencing are small interfering RNA (siRNA) and microRNA

\footnotetext{
* Correspondence: mqw@hvri.ac.cn

State Key Laboratory of Veterinary Biotechnology, Harbin Veterinary Research Institute, Chinese Academy of Agricultural Sciences, No.427 Maduan Street, Nangang District, Harbin 150001, People's Republic of China
}

(miRNA), where the siRNAs mediate specific mRNA degradation, whereas miRNA inhibits specific mRNA at the translational level. Both of these biological processes are considered key methods of modulating host gene expression, and these two molecules are also involved in antiviral and transposon silencing pathways.

The RNAi strategy has been successfully applied to the inhibition of viral replication. It has been demonstrated that some genes inhibited by siRNAs, such as $p 24$, vif, nef, tat, and rev, can block Human immunodeficiency virus (HIV) replication in cells [2]. The infection of cells by HIV may be hindered by inhibiting the expression of the HIV receptors CD4 and CD8a, their coreceptors CXCR4 or CCR5, or the virus Gag structural protein [3]. In some studies, transfection of siRNA designed to target $\mathrm{C}$ virus (HCV) remarkably inhibited the expression of virus-specific proteins and protected cells against HCV RNA, in vitro [4,5]. In another study, Hepatitis B virus (HBV) replication was successfully inhibited after plasmid expression of HBV siRNA transfected into mouse liver [6]. Hu et al. [7] adopted siRNA designed against the ALV gag gene and demonstrated 
significantly reduced virus replication. Chen et al. [8] indicated that ALV-B replication was significantly inhibited after knockdown of the ALV-B tvb and env genes.

Although siRNAs have been widely used as gene-silencing molecules, the intrinsic drawbacks of siRNA methodology have been revealed. Off-target effects may be produced where siRNA function must be fully and completely complementary to the target sequence; if the virus mutates, siRNA will produce off-target effects. Other drawbacks include the elicitation of the interferon response and interference with endogenous miRNA biogenesis. The unique biogenesis and mechanism of action of miRNA do reduce the likelihood of these problems.

Despite these problems, the RNAi strategy remains an attractive option for antiviral therapy and for the functional analysis of genes for several reasons. First, RNAi has sequence specificity. Second, the application of multi-series RNAi can target different genes or sequences simultaneously and hence can minimize the possibility of the virus acquiring mutations that confer resistance. Third, RNAi can be transmitted from nonpathogenic viruses to pathogenic viruses. Finally, due to its sequence specificity, siRNA designed against a virus can only inhibit that virus, leaving vaccine strains unaffected. To ensure a high level of RNAi gene silencing, multiple miRNAs can be designed. These can be transfected into cells using the same transfer medium, and by targeting different sequences, mutation of the target virus, and therefore the probability of evasion from the silencing effect of the miRNA is minimized.

ALV, especially ALV-J, brings about enormous economic losses in the poultry industry. The virus has rapidly spread worldwide and transmits both vertically and horizontally. However, chicks are immunologically tolerant to ALV infection. Unfortunately, to date, no effective vaccine has been developed against ALV. In this study, we used RNAi technology to inhibit ALV replication and screened the effective target sites for their ability to inhibit ALV replication at a cellular level. Our findings could pave the way for anti-ALV gene screening and the development of disease resistance.

\section{Materials and methods}

\section{Viruses and cells}

The SD strains of ALV-J were isolated from poultry in Shandong, China, and stored at the Harbin Veterinary Research Institute (Harbin, China), a Key State Laboratory. The DF-1 cell lines [9] were provided by Zhigao $\mathrm{Bu}$ at the Harbin Veterinary Research Institute (Harbin, China).

\section{Reagents}

The linearized the pcDNA6.2-GW/EmGFP-miR eukaryotic expression vector, Escherichia coli TOP10 cells and the Lipofectamine 2000 transfection reagent were purchased from Invitrogen (Carlsbad, CA, USA). Ligase and reverse transcriptase were purchased from TaKaRa (Dalian, China). The fluorescence quantitative PCR kit was purchased from BIOER Technology (Hangzhou, China). The plasmid extraction kit and the viral RNA extraction kit were purchased from Shanghai Watson Biotech (Shanghai, China). The goat anti-mouse IgG/ fluorescein isothiocyanate (FITC) antibody and the horseradish peroxidase-labeled goat anti-mouse IgG were purchased from Zhongshan Goldbridge Biotechnology (Beijing, China). The anti-ALV-J monoclonal antibody JE-9 was kindly provided by Professor Qin Aijian (Yangzhou University, China). All other chemicals were of analytical reagent grade.

\section{Design and construction of miRNA expression vector Design of miRNA}

Four pairs of miRNAs sequences were designed against the conserved regions of the gag gene (NC-015116 gag) using online software http://rnaidesigner.invitrogen.com/ rnaiexpress/ (Table 1). Designed miRNA sequences were synthesized by Shanghai Health Bioengineering (China). Double-stranded oligonucleotide encoding pre-miRNA sequence were annealed and inserted into the linearized expression vector, pcDNA6.2-GW/EmGFP-miR (5 ng/ $\mu \mathrm{L})$, to construct recombinant plasmids containing the target miRNAs, designated mi-gag1318, mi-gag1365, migag1623, mi-gag1971. All recombinant plasmids have been sequenced to confirm the sequences inserted.

\section{Construction of miRNA expression vectors in series}

Two tandem plasmids were constructed based on the migag1318 plasmid backbone. After BglI and XhoI digestion of mi-gag1318, the miRNA sequence of the pol gene (NC015116 pol), mi-pol2516 (constructed previously, manuscript submitted), was digested with SalI and BglI, and ligated to mi-gag1318. The tandem plasmid consisting of mi-gag1318 and mi-pol2516 was designated mi-g1318p2516. The mi-g1318-e1384 (env gene: NC-015116 env) and mi-p2516-e1384 plasmids were constructed in the same way. Plasmids were linearized and used to transform E. coli TOP10. Positive clones were selected and verified by digestion with XhoI and SalI and sequencing. Similarly, based on the recombinant plasmid mi-g1318-p2516 backbone, a tandem plasmid consisting of mi-gag1318, mipol2516 and mi-env1384 was constructed in the same way and designated mi-g1318-p2516-e1384. Positive clones were verified by double digestion with XhoI and SalI and sequencing.

\section{Transfection of recombinant plasmid and preparation of ALV}

DF-1 cells were cultured in Dulbecco's Modified Eagle's Medium (DMEM) containing $100 \mathrm{U} / \mathrm{mL}$ of penicillin 
Table 1 Oligonucleotide sequences of pre-miRNAs

\begin{tabular}{|c|c|}
\hline & Sequence $\left(5^{\prime}\right.$ to $\left.3^{\prime}\right)$ \\
\hline \multirow[t]{2}{*}{ mi-gag1318 } & TGCTGTTGATCACAAGACTGGCTGATGTITGGCCACTGACTGACATCAGCCACTTGTGATCAA \\
\hline & CCTGTTGATCACAAGTGGCTGATGTCAGTCAGTGGCCAAAACATCAGCCAGTCTTGTGATCAAC \\
\hline \multirow[t]{2}{*}{ mi-gag1365 } & TGCTGTAGTGATTAAGACAGAGGGACGTTITGGCCACTGACTGACGTCCCTCTCTTAATCACTA \\
\hline & CCTGTAGTGATTAAGAGAGGGACGTCAGTCAGTGGCCAAAACGTCCCTCTGTCTTAATCACTAC \\
\hline \multirow[t]{2}{*}{ mi-gag1623 } & TGCTGATCATTGCGGAACAGCTATTGGTTTGGCCACTGACTGACCAATAGCTTCCGCAATGAT \\
\hline & CCTGATCATTGCGGAAGCTATTGGTCAGTCAGTGGCCAAAACCAATAGCTGTTCCGCAATGATC \\
\hline \multirow[t]{2}{*}{ mi-gag1971 } & TGCTGTTATGTCTCCCTCAGACTTATGTTITGGCCACTGACTGACATAAGTCTGGGAGACATAA \\
\hline & CCTGTTATGTCTCCCAGACTTATGCAGTCAGTGGCCAAAACATAAGTCTGAGGGAGACATAAC \\
\hline
\end{tabular}

and streptomycin, and containing $5 \%(\mathrm{v} / \mathrm{v})$ fetal calf serum (PAA GOLD, Austria). Twenty-four hours before transfection, cells that had grown to $70 \%$ confluence were used to inoculate cell culture plates at a density of 3-5 $\times 10^{5}$. When the cells had grown to $80 \%$ confluence, they were transfected with the RNAi expression plasmid using Lipofectamine 2000. Six hours later, the culture fluid was changed DMEM and serum containing 5\% fetal calf serum, without antibiotics. After incubation at $37^{\circ} \mathrm{C}$ and $5 \% \mathrm{CO}_{2}$ for $12 \mathrm{~h}$, the transfection efficiency was determined by the expression of green fluorescent protein observed using fluorescence microscopy. Transfected cells were inoculated with $10050 \%$ tissue culture infectious doses $\left(\mathrm{TCID}_{50}\right)$ of ALV-J. The culture medium was obtained $72 \mathrm{~h}$ after viral infection, and the inhibitory effect was detected by an indirect immunofluorescence assay (IFA), western blotting, and real-time PCR. In this study, the negative control group that was not administered plasmid, the null vector control group and the test groups were randomized for analysis.

\section{Determination of ALV-J titer}

Strain SD was inoculated into DF-1 cell culture bottles, freeze-thawed three times and then centrifuged at 7000 $\times g$ and $4^{\circ} \mathrm{C}$ for $5 \mathrm{~min}$. The supernatant was collected and 10 -fold serially diluted to $10^{-8}$ and then inoculated into 96-well plates along with DF-1 cells. Four parallel well were created in each gradient. After incubation for 5 days, cells were fixed by cooling in methanol at $-20^{\circ} \mathrm{C}$ for $30 \mathrm{~min}$, and then washed three times with phosphate buffered saline containing 0.05\% Tween 20 (PBST) for 10 min each. ALV-J specific monoclonal antibody JE-9 was diluted 1:200 and $100 \mu \mathrm{L}$ was added to each well. The cells were incubated at $37^{\circ} \mathrm{C}$ for $1 \mathrm{~h}$ and washed with PBST three times, for $10 \mathrm{~min}$ each. The secondary antibody, goat anti-mouse IgG/FITC, was added at a dilution of 1:200, and the cells were incubated at $37^{\circ} \mathrm{C}$ for $45 \mathrm{~min}$ away from the light. The cells were then washed with PBST five times, for $10 \mathrm{~min}$ each. The number of fluorescent cells per well were counted using a fluorescence microscope, and the $\mathrm{TCID}_{50}$ was calculated using the Reed-Muench method [10]. Therefore, the toxic potency of the virus was determined to be $10^{5.2} \mathrm{TCID}_{50} / \mathrm{mL}$.

\section{Expression of the envelope glycoprotein by IFA}

Virus strain SD was used to inoculate DF-1 cells transfected with recombinant plasmid using the method described above. After 5 days incubation, cells were treated with the fluorescent secondary antibody, rhodamine-labeled goat anti-mouse IgG/TRITC, as described above. The results were photographed using a fluorescence microscopy.

\section{Western blot}

At about $72 \mathrm{~h}$ after transfection with the miRNA recombinant plasmid, DF-1 cells were collected and the residual supernatant fluid was washed with PBS. Total protein was extracted using the total protein extraction kit (BestBio, China) and quantitated using a spectrophotometer. Sodium dodecyl sulfate-polyacrylamide gel electrophoresis (SDS-PAGE; 10\%) was performed using a sample volume of $300 \mu \mathrm{g}$ per well. Samples were transferred to nitrocellulose membrane after separation, blocked at $4^{\circ} \mathrm{C}$ overnight and then washed with PBST three times, for 10 min each. ALV-J-specific monoclonal antibody JE-9 was added and the cells were incubated at $37^{\circ} \mathrm{C}$ for $1 \mathrm{~h}$ and mouse anti-chicken glyceraldehyde-3-phosphate dehydrogenase (GAPDH) monoclonal antibodies were added and incubated at room temperature for $1 \mathrm{~h}$, followed by three washes with PBST for 10 min each. Horseradish peroxidase-labeled anti-mouse secondary antibody was added and cells were incubated at $37^{\circ} \mathrm{C}$ for $45 \mathrm{~min}$. After washing with PBST five times, 10 min each wash, the cells were stained with 3,3' diaminobenzidine (DAB, 6 $\mathrm{mg}, 10 \mathrm{~mL}$ TBS, $0.1 \mathrm{~mL} 3 \% \mathrm{H}_{2} \mathrm{O}_{2}$ ) and then scanned using the gel imaging system AlphaImager HP.

\section{Quantitative PCR}

Using sequences of the ALV gag genes published in the GenBank database (NC-015116), pairs of primers were designed using the Oligo 6 primer design software to 
amplify the conserved regions of the gene. Additionally, a pair of ALV-J specific primers was also synthesized. Two pairs of target genes and the $\beta$-actin gene were amplified by PCR using the SD cDNA as a template (Table 2).

The cDNAs amplified using the gag, ALV-specific, and $\beta$-actin primers were cloned into vector pMD-18-T and used to transform competent E. coli DH5 $\alpha$ cells. Positive clones were isolated and verified by colony PCR and sequencing, and designated pMT-G and pMT-ALV, respectively. The concentration and purity of verified plasmids were determined according to the formula: copy number $=($ mass $/$ molecular weight $) \times 6.0 \times 10^{23}$. Extracted plasmids were then ten-fold serially diluted and used as temples for quantitative PCR amplification and delineation of quantitative PCR standard curves.

Total RNA was extracted from viruses and cells $72 \mathrm{~h}$ after the infection of DF-1 cells with ALV-J using Trizol, according to the manufacturer's instructions. RNA concentration and purity was determined by measuring optical density (OD) at wavelengths of 260 and 280 $\mathrm{nm}$ using a standard spectrophotometer. The $\mathrm{OD}_{260} /$ $\mathrm{OD}_{280}$ ratios were more than 1.8 for all samples. Quantitative PCR was performed in a LightCycler 480 Real-Time PCR System, for the gag and ALV-specific genes using the SYBR GREEN kit (SYBR ${ }^{\circledR}$ Premix Ex Taq ${ }^{\mathrm{TM}}, \mathrm{TaKaRa}$, Dalian, China) and the primers are listed in Table 1. Amplification was carried out in a 20 $\mu \mathrm{l}$ reaction mixture containing $10 \mu \mathrm{lSYBR}^{\circledR}$ Premix Ex $\mathrm{Taq}^{\mathrm{TM}} 2 \times, 0.2 \mu \mathrm{M}$ concentration of each primer, $1 \mu \mathrm{l}$ cDNA. The reaction procedure was $95^{\circ} \mathrm{C} 10 \mathrm{~s}$, followed by 40 cycles at $95^{\circ} \mathrm{Cfor} 5 \mathrm{~s}$ and $60^{\circ} \mathrm{C}$ for $40 \mathrm{~s}$. The number of target genes in different samples was determined according to the standard curve after the reaction. Meanwhile, the copy number of the $\beta$-actin gene was also determined by quantitative PCR. To confirm specific amplification, melting curve analysis was performed.

\section{Data analysis}

Experiments were repeated three times and values were expressed as means \pm standard deviation (SD). The $t$ test was performed using the SPSS 13.0 statistical software (version 13.0; SPSS, USA). Differences were considered statistically significant when $p<0.05$.

\section{Results \\ Envelope protein expression}

As shown in Figure 1a and 1b, IFA revealed that the recombinant plasmids, mi-gag1318 and mi-gag1365, significantly reduced the fluorescence intensity. The tandem plasmids, mi-g1318-p2516, mi-g1318-e1384, mip2516-e1384 and mi-g1318-p2516-e1384, also significantly inhibited the fluorescence intensity (Figure 2a and $2 \mathrm{~b}$ ) compared with the negative control group. However, no significant differences were detected among other groups compared with the negative control group and the null vector group.

Western blot analysis of the ALV-J envelope glycoprotein JE-9 is an anti-ALV-J envelope glycoprotein monoclonal antibody that specifically identifies a protein with a molecular weight of 90-94 $\mathrm{kDa}$. As detected by western blot analysis (Figure 3a, b), the recombinant mi-gag1318 and mi-gag1365 plasmids decreased ALV-J envelope glycoprotein expression significantly, while there were no significant differences determined between the remaining groups and the negative control or null vector groups. Transfection of the tandem plasmids into DF-1 cells significantly decreased the expression of the ALV-J envelope glycoprotein, indicating that ALV-J could inhibit plasmid replication.

\section{Quantitative PCR of ALV-J mRNA}

Expression of the gag gene in cells transfected with plasmids mi-gag1318 and mi-gag1365 differed significantly from the negative control group and the null vector control group $(P<0.05)$, while expression in cells transfected with plasmids mi-gag1623 and mi-gag1971 did not differ significantly from that in the negative control and null vector control groups $(P>0.05)$ (Table 3$)$. The use of tandem plasmids indicated significant inhibition of the expression of the ALV-J envelope glycoprotein in DF-1 cells (85-91.2\%), and the rate of inhibition was highest with plasmid mi-g1318-p2516-e1384 (91.2\%) (Table 4).

Table 2 Primers used to amplify target genes

\begin{tabular}{|c|c|c|c|}
\hline Target gene (Accession number) & Primer sequence & Product size (bp) & Annealing Temperature $\left({ }^{\circ} \mathrm{e}\right)$ \\
\hline \multirow[t]{2}{*}{ Gag } & Forward TCAGGACCAAGGGCTTAC & 174 & 55.2 \\
\hline & Reverse CTGCCGCTATAACCGTCTG & & \\
\hline \multirow[t]{2}{*}{ ALV specific primer } & Forward TCAGGACCAAGGGCTTAC & 545 & 55.0 \\
\hline & Reverse CTGCCGCTATAACCGTCTG & & \\
\hline \multirow[t]{2}{*}{$\beta$-actin } & Forward TCCCTGTATGCCTCTGGTC & 250 & 55.0 \\
\hline & Reverse TCTCTCTCGGCTGTGGTGG & & \\
\hline
\end{tabular}




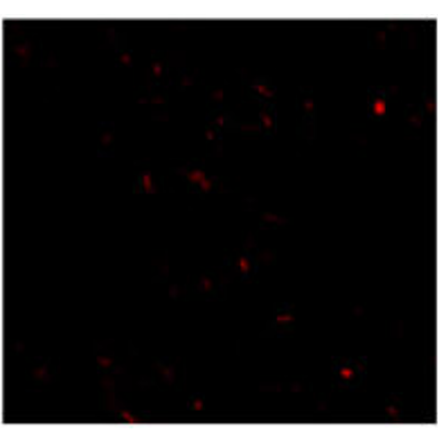

mi-gag 1318

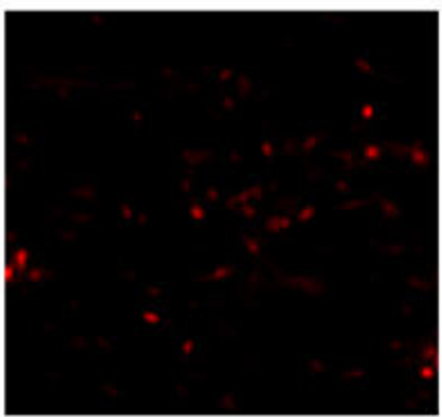

Fig 1.A

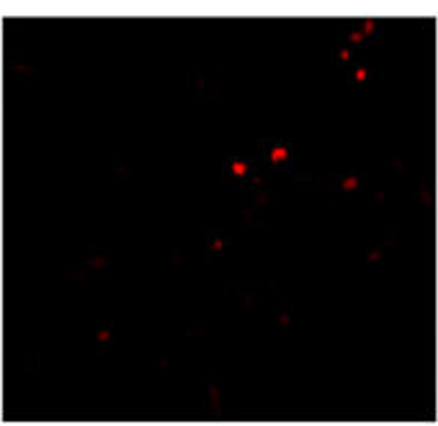

mi-gag 1365

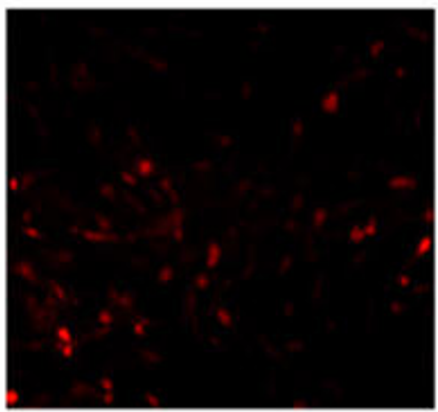

Negative control

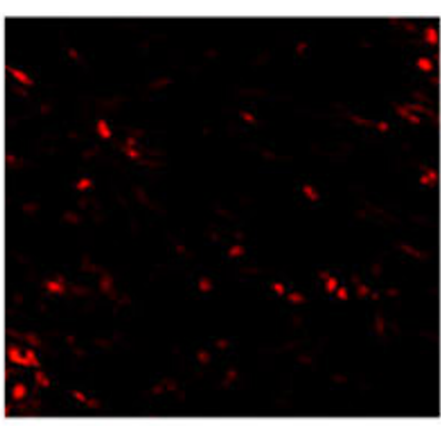

mi-gag 1623

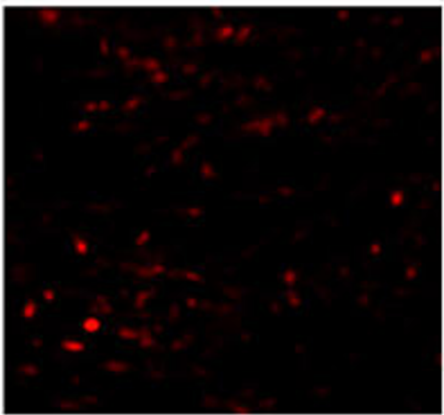

Null-vector control

Fig 1.B

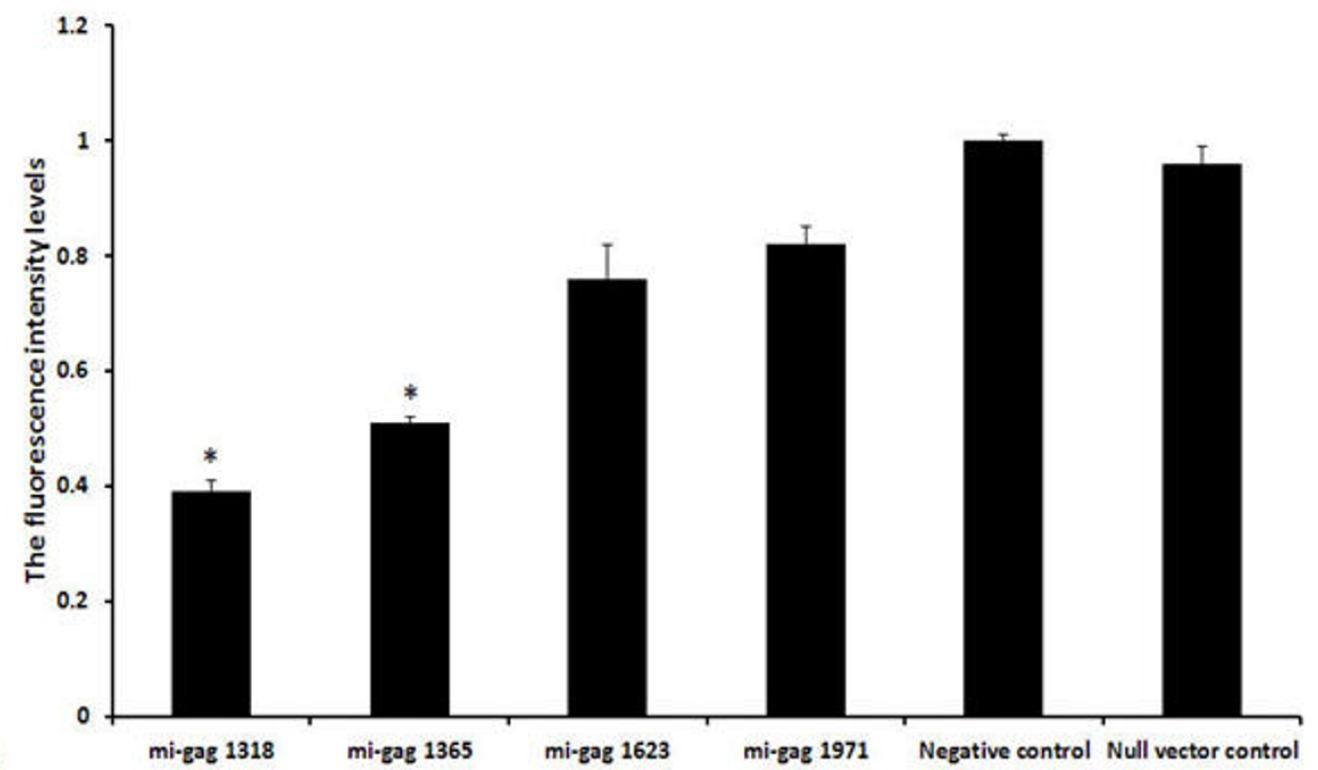

Figure 1 ALV-J replication. (A) Cells transfected with the recombinant plasmid pMD-G and their inhibitory effects against ALV-J, as determined by the IFA. (B) The fluorescence intensity of cells transfected with the recombinant plasmid pMD-G and their inhibitory effects against ALV-J, as determined by the IFA. Data are presented as means \pm S.E.M. of three independent experiments, each performed in triplicate. *Statistically significant differences compared with negative controls $(P<0.05)$. 


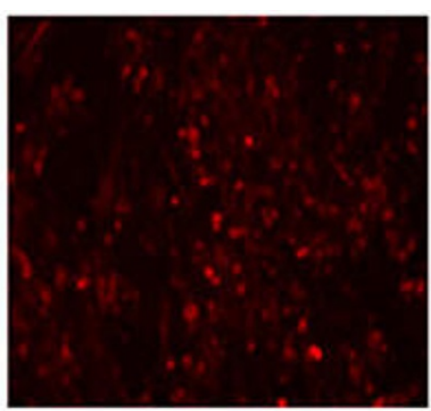

Negative control

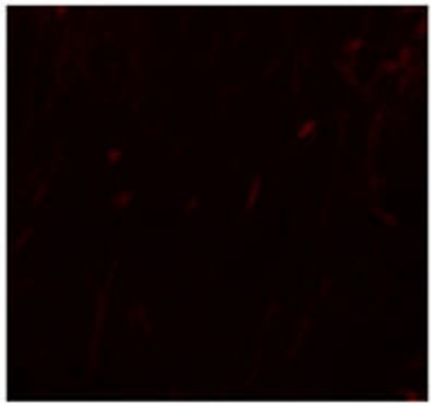

mi-g1318-p2516

Fig 2.A
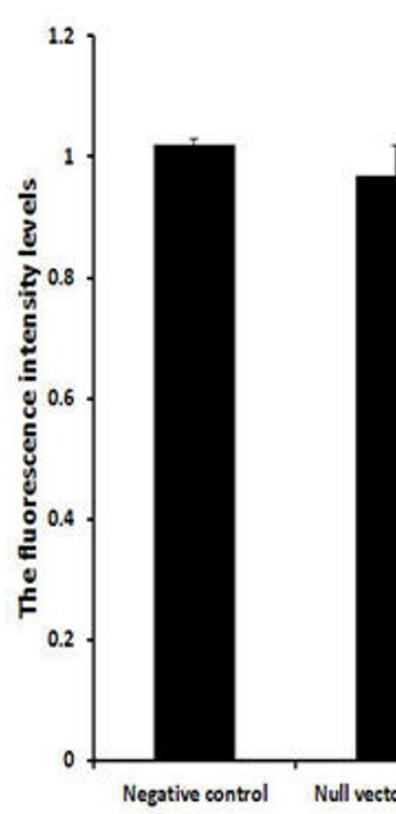

Fig 2.B

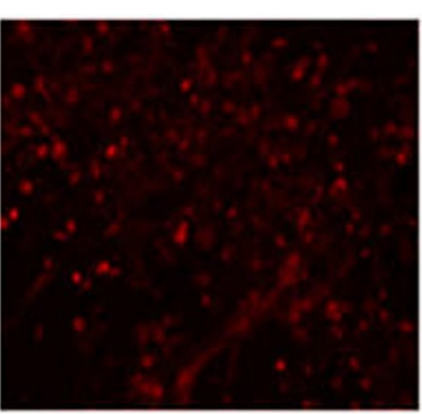

Null-vector control

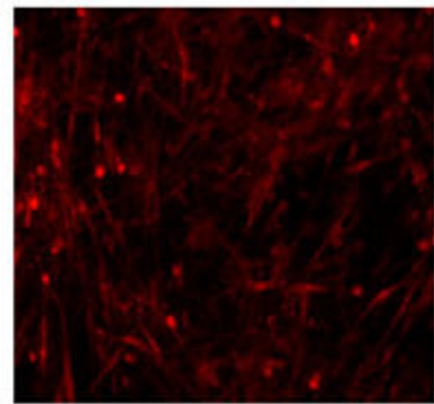

mi-g1318-e1384

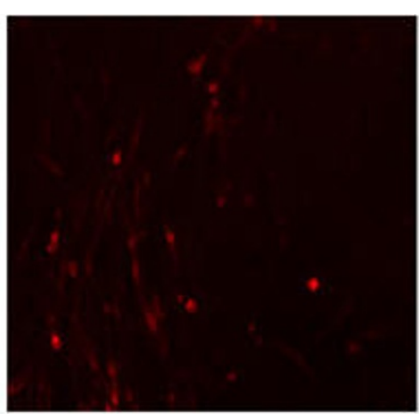

mi-p2516-e1384

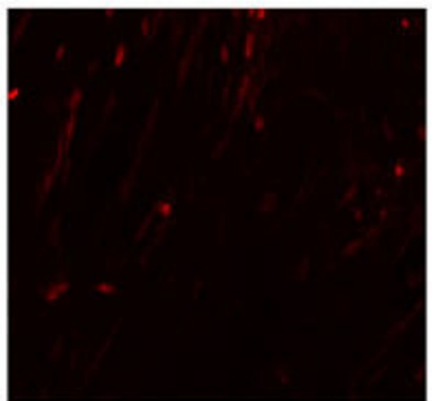

mi-gag1318-p2516-e1384

Figure 2 Inhibition of ALV-J replication by miRNA. (A) Cells transfected with the recombinant serial miRNA plasmids and their inhibitory effects against ALV-J, as determined by the IFA. (B) The fluorescence intensity of cells transfected with the recombinant serial miRNA plasmids and their inhibitory effects against ALV-J, as determined by the IFA. Data are presented as means \pm S.E.M. of three independent experiments, each performed with triplicate samples. *Statistically significant differences compared with negative controls $(P<0.05)$. 


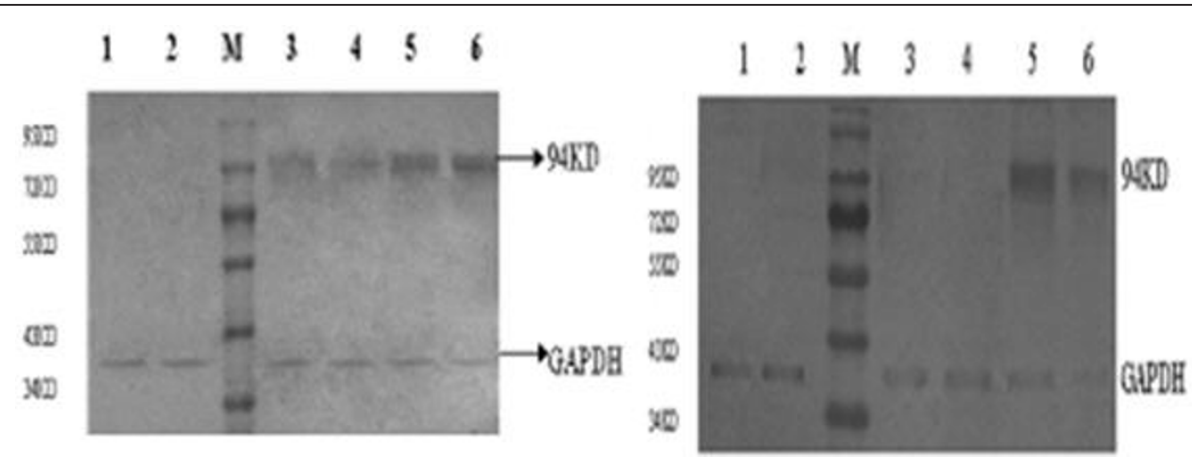

Figure 3 ALV-J envelope glycoprotein expression of DF-1 cells in each in of the groups. (A) Western immunoblot of infected culture treated with miRNA. Lane M: protein ladder; lane 1: mi-gag1318; lane 2: mi-gag1356; lane 3: mi-gag1623; lane 4: mi-gag1971; lane 5: negative control; lane 6: null vector control. (B) Western immunoblot of infected cultures treated with miRNA. Lane M: protein ladder; lane 1: mi-g1318e1384; lane 2: mi-p2516-e1384; lane 3: mi-g1318-p2516; lane 4: mi-g1318-p2516-e1384; lane 5: negative control; lane 6: null vector control.

\section{Discussion}

Accumulating evidence suggests that RNAi can inhibit viral replication in vivo and in vitro. RNAi technology have been applied in numerous studies including those for HBV [11], HCV [12], HIV-1 [13], influenza virus A [14], aphthovirus of cattle $[15,16]$, and severe acute respiratory syndrome (SARS) virus [17] infections. RNAi has been used to suppress the replication of herpesviruses, including Murine herpesvirus 68 [18], Herpes simplex virus-1 [19], Human cytomegalovirus [20], and Duck herpesvirus [21].

The ALV-J genome contains a gene arrangement of LTR-leader-gag-pol-env-LTR. The gag and pol genes are highly conserved, sharing 96-97\% homology in ALV-J, in contrast to subgroups $\mathrm{A}, \mathrm{C}$, and $\mathrm{D}$. The pol gene mainly encodes the reverse transcriptase (RT; P68) and viral integrase (IN; P32). RT is responsible for the production of proviral DNA using viral RNA as a template, while IN is involved in the integration of proviral DNA into the host genome. The pol gene is necessary for reverse transcription of the RNA genome and the generation of viral DNA, and it is a key to the insertion of the viral genome into the host genome. RNAi has been applied to the inhibition of ALV replication by a number of research groups. Chen et al. [8] successfully inhibited ALV-B replication induced by a retroviral vector by targeting the miRNA of the ALV-B env gene and its receptor encoded by the $t v b$ gene.

By constructed an miRNA expression vector targeting the ALV-J gag gene, we demonstrated that mi-gag1318 and mi-gag1365 could significantly reduce the expression of target gene mRNA and envelope glycoprotein at a cellular level, with the highest inhibition rate of $77.3 \%$ being observed with mi-gag1318. These results showed that miRNA expression could inhibit the duplication of the target gag gene, with mi-gag1318 having the highest inhibitory effect. Accordingly, the miRNA of the gag target genes could successfully inhibit ALV-J replication. Thus, the successful construction of a eukaryotic expression vector would contribute to the selection and propagation of an anti-ALV related gene.

In previous studies, RNAi strategies have been successfully employed experimentally to inhibit virus replication. Hu et al. [7] showed that by electroporation into chicken embryos, siRNA containing ALV gag sequences effectively slowed down virus propagation. In the current study, the miRNA target gag1318 was selected that resulted in significant inhibitory effects on ALV-J replication. These miRNAs were grouped in pairs or together, and the tandem multi-target miRNAs target

Table 3 Quantitative PCR of the miRNA-targeted gag gene inhibiting ALV-J

\begin{tabular}{|c|c|c|c|c|c|}
\hline Group & $\begin{array}{l}\text { mRNA levels of } \\
\text { gag }\end{array}$ & $\begin{array}{l}\text { mRNA levels of } \beta \text { - } \\
\text { actin }\end{array}$ & $\begin{array}{l}\text { mRNA levels of target gene by } \beta \text {-actin } \\
\text { normalization }\end{array}$ & $\begin{array}{c}\text { Rates of viral suppression } \\
(\%)\end{array}$ & $\begin{array}{c}\mathrm{p}- \\
\text { values }\end{array}$ \\
\hline mi-gag1318 & $1.05 \pm 0.11$ & $3.21 \pm 0.12$ & 0.327 & $77.3^{*}$ & 0.01 \\
\hline mi-gag 1365 & $1.10 \pm 0.12$ & $3.14 \pm 0.15$ & 0.3350 & $65.0^{*}$ & 0.02 \\
\hline mi-gag 1623 & $2.81 \pm 0.11$ & $3.49 \pm 0.16$ & 0.805 & 19.5 & 0.06 \\
\hline mi-gag 1971 & $3.12 \pm 0.17$ & $3.85 \pm 0.16$ & 0.810 & 19.0 & 0.07 \\
\hline $\begin{array}{l}\text { Negative } \\
\text { control }\end{array}$ & $3.15 \pm 0.12$ & $3.14 \pm 0.12$ & 1.00 & 0 & 0 \\
\hline Vector control & $3.62 \pm 0.14$ & $3.70 \pm 0.12$ & 0.978 & 0 & 0 \\
\hline
\end{tabular}


Table 4 Quantitative PCR of the target miRNA inhibiting ALV-J replication

\begin{tabular}{|c|c|c|c|c|c|}
\hline Group & $\begin{array}{l}\text { mRNA levels of target } \\
\text { gene }\end{array}$ & $\begin{array}{l}\text { mRNA levels of } \beta \text { - } \\
\text { actin }\end{array}$ & $\begin{array}{c}\text { mRNA levels of target gene by } \beta \text {-actin } \\
\text { normalization }\end{array}$ & $\begin{array}{c}\text { Rates of viral } \\
\text { suppression (\%) }\end{array}$ & $\begin{array}{c}p- \\
\text { values }\end{array}$ \\
\hline mi-g1318-p2516 & $0.62 \pm 0.17$ & $4.59 \pm 0.16$ & 0.135 & $86.5^{*}$ & 0.01 \\
\hline mi-g1318-e1384 & $0.58 \pm 0.11$ & $3.87 \pm 0.13$ & 0.150 & $85.0^{*}$ & 0.01 \\
\hline mi-p2516-e1384 & $0.48 \pm 0.10$ & $4.02 \pm 0.15$ & 0.120 & $88.0^{*}$ & 0.01 \\
\hline $\begin{array}{l}\text { mi-g1318-p2516- } \\
\text { e1384 }\end{array}$ & $0.35 \pm 0.09$ & $3.98 \pm 0.12$ & 0.088 & $91.2^{*}$ & 0.01 \\
\hline Negative control & $3.75 \pm 0.18$ & $3.68 \pm 0.15$ & 1.030 & 0 & 0 \\
\hline Vector control & $3.18 \pm 0.12$ & $3.25 \pm 0.10$ & 0.987 & 0 & 0 \\
\hline
\end{tabular}

Statistically significant differences from the Negative control are indicated by* $(P<0.05)$

mi-g1318-p2516, mi-g1318-e1384, mi-p2516-e1384, and mi-g1318-p2516-e1384 were co-expressed, reducing the probability of evasion from the silencing effect of the miRNA due to virus mutation. When multiple miRNAs were expressed simultaneously, gene silencing was shown to be more effective. Tandem plasmids were transfected into DF-1 cells and then infected with ALVJ. IFA, western blotting, and quantitative PCR were used to evaluate the inhibiting effects on ALV-J replication at the cellular level. The results verified that all of the tandem plasmids could effectively inhibit the replication of ALV in DF-1 cells, with an inhibition efficiency of 8591.2\%. The enhanced inhibitory effects conferred by the multi-target miRNA expression plasmids demonstrated that the multi-target miRNAs could inhibit ALV synergistically.

These studies successfully identified targets capable of inhibiting the replication of ALV-J and are likely to be good candidates for the development of miRNA-based vaccines. The current study showed that the strategy of using multi-target miRNAs might be an effective method for inhibiting viral replication and for the acquisition of resistant mutations.

\section{Acknowledgements \\ This work is supported by the Ministry of Agriculture of China (2009ZX08006-001B); National Natural Science Foundation of China (30771615); National Science and Technology Support Program during the Eleventh Five-Year Plan Period (2006BDA13B08-17)}

\section{Authors' contributions}

QWM conceived the study, participated in its design and coordination, and finalized the manuscript in its final form. ZPZ performed the experiments. WW carried out the statistical analyses and participated in drafting of the manuscript. JT carried out the replication studies. ZGX carried out the quantitative PCR. All authors read and approved the final manuscript.

\section{Competing interests}

The authors declare that they have no competing interests.

Received: 8 June 2011 Accepted: 22 December 2011 Published: 22 December 2011

\section{References}

1. Zhang L-J, Liu J, Cheng Z-Q, Wang G-H, Diao X-G, Zhu G: Detecting avian leukosis virus subgroup $J$ using tissue chip and immunohistochemical technology. Chin J Prevent Vet Med 2006, 4:427-430.

2. Nielsen MH, Pedersen FS, Kjims J: Molecular strategies to inhibit HIV-1 replication. Retrovirology 2005, 2:10

3. Lee NS, Dohjima T, Bauer G, Li G, Li MJ, Ehsani A, Salvaterra P, Rossi J: Expression of small interfering RNAs targeted against HIV21 rev transcripts in human cells. Nat Biotechnol 2002, 20:500-505.

4. Sagan SM, Nasheri N, Luebbert C, Pezacki JP: The efficacy of siRNA against hepatitis $C$ virus is Strongly influenced by structure and target site accessibility. Chem Biol 2010, 17:515-27.

5. Christophe C, Saulnier A, Benureau Y, Flechet D, Delgrange D, ColbereGarapin F, Wychowski C, Martin A: Inhibition of hepatitis C virus infection in cell culture by small interfering RNAs. Mol Ther 2007, 15(8):1452-1462.

6. McCaff rey AP, Nakai H, Pandey K, Huang Z, Salazar FH, Xu H, Wieland SF, Marion PL, Kay MA: Inhibition of hepatitis $B$ virus in mice by RNA interference. Nat Biotechnol 2003, 21:639-644.

7. Hu WY, Myers CP, Kilzer JM, Pfaff SL, Bushman FD: Inhibition of retroviral pathogenesis by RNA interference. Curr Biol 2002, 12(15):1301-1311.

8. Chen M, Granger AJ, VanBrocklin MW: Inhibition of avian leukosis virus replication by vector-based RNA interference. Virology 2007, 365:464-472

9. Himly M, Foster DN, Bottoli I, lacovoni JS, Vogt PK: The DF1 chicken fibroblast cell liner: transformation induced by diverse oncogene and cell death resulting from infection by avian leukosis viruses. Virology 1998, 248:295-304.

10. Reed $L$, Muench $\mathrm{H}$ : A simple method of estimating fifty percent endpoints. Am J Hygiene 1938, 27(3):493-497.

11. Wu K, Mu Y, Hu J, Lu L, Zhang X, Yang Y, Li Y, Liu F, Song D, Zhu Y, Wu J: Simultaneously inhibition of HIV and HBV replication through a dual small interfering RNA expression system. Antiviral Res 2007, 74(2):142-149.

12. Randall G, Rice CM: Interfering with hepatitis $C$ virus RNA replication. Virus Res 2004, 102:19-25.

13. Coburn GA, Cullen BR: Potent and specific inhibition of human immunodeficiency virus type 1 replication by RNA interference. J Virol 2002, 76(18):9225-31

14. Ge Q, McManus MT, Nguyen $T$, Shen $C H$, Sharp PA, Eisen HN, Chen J: RNA interference of influenza virus production by directly targeting mRNA for degradation and indirectly inhibiting all viral RNA transcriptioin. Proc Natl Acad Sci USA 2003, 100:2718-2723.

15. Chen W, Yan W, Du Q, Fei L, Liu M, Ni Z, Sheng Z, Zheng Z: RNA interference targeting VP1 inhibits foot-and mouth disease virus replication in BHK-21 cells and suckling mice. J Virol 2004, 78:6900-6907.

16. Liu M, Chen W, Ni Z, Yan W, Fei L, Jiao Y, Zhang J, Du Q, Wei X, Chen J, Liu Y, Zheng Z: Cross-inhibition to heterologous foot-and-mouth disease virus infection induced by RNA interference targeting the conserved regions of viral genome. Virology 2005, 336:51-59.

17. Lu A, Zhang H, Zhang X, Wang H, Hu Q, Shen L, Schaffhausen BS, Hou W, Li L: Attenuation of SARS coronavirus by a short hairpin RNA expression plasmid targeting RNA-dependent RNA polymerase. Virology 2004, 324(1):84-89. 
18. Jia $Q$, Sun R: Inhibition of gammaherpesvirus replication by RNA interference. J Virol 2003, 77:3301-3306.

19. Bhuyan PK, Kariko K, Capodici J, Lubinski J, Hook LM, Friedman HM, Weissman D: Short interfering RNA-mediated inhibition of herpes simplex virus type 1 gene expression and function during infection of herpes simplex virus type 1 gene expression and function during infection of human keratinocytes. J Virol 2004, 78:10276-10281.

20. Wiebusch L, Truss M, Hagemeier C: Inhibition of human cytomegalovirus replication by small interfering RNAs. J Gen Virol 2004, 85:179-184.

21. Mallanna SK, Rasool TJ, Sahay B, Aleyas AG, Ram H, Mondal B, Nautiyal B, Premrai A, Sreekumar E, Yadav MP: Inhibition of Anatid Herpes Virus-1 replication by small interfering RNAs in cell culture system. Virus Res 2006, 115:192-197.

doi:10.1186/1743-422X-8-556

Cite this article as: Meng et al:: Enhanced inhibition of Avian leukosis virus subgroup J replication by multi-target miRNAs. Virology Journal $20118: 556$

\section{Submit your next manuscript to BioMed Central} and take full advantage of:

- Convenient online submission

- Thorough peer review

- No space constraints or color figure charges

- Immediate publication on acceptance

- Inclusion in PubMed, CAS, Scopus and Google Scholar

- Research which is freely available for redistribution

Submit your manuscript at www.biomedcentral.com/submit 